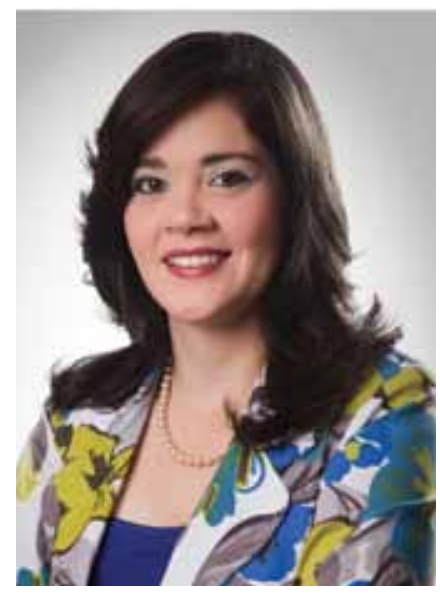

ECOS DESDE LAS FACULTADES

\title{
Estudios Generales: el qué y el para qué. Apuntes para una reflexión
}

\author{
Luisa Taveras ${ }^{*}$ y Rosario Olivo ${ }^{*}$
}

Resumen: Este artículo se escribe desde el Departamento de Ciclo Básico de la Pontificia Universidad Católica Madre y Maestra, con el objetivo de que las ideas aquí expuestas sean un aporte a la reforma del Ciclo Básico de esta Institución, que se encuentra en proceso de reflexión y revisión. Se expone el concepto de Estudios Generales desde la óptica de varios destacados pensadores y académicos en Educación Superior. Asimismo, se hace énfasis en que el fin principal de la Institución es lograr en sus egresados una formación integral, donde converjan conocimientos, habilidades, actitudes y valores, fundamentados en el humanismo cristiano.

\begin{abstract}
By means of this article, the PUCMM Department of Basic Education Term wishes to contribute to the reform of this Institution's Basic Education Term, which is currently undergoing a process of reflection and revision, with a collection of ideas that are presented here. These ideas discuss the concept of General Studies from the point of view of prominent thinkers and scholars in the area of Higher Education. It lays stress on the fact that the mission of the PUCMM is to have their graduates achieve a comprehensive education, one that encompasses abilities, attitudes and values based on Christian humanism.
\end{abstract}

En este mundo lleno de diferencias y contradicciones uno de los puntos en que la gran mayoría está de acuerdo es que la educación transforma al individuo y, por ende, a la sociedad. Esta mayoría reconoce que la educación es el elemento fundamental si queremos alcanzar una sociedad con calidad de vida, desarrollo sostenible, democracia participativa y equidad; en palabras de Kant, una sociedad ilustrada que salga de su minoría de edad.

Por esta razón, estamos convencidos de que la educación tiene una función social, ya que participa en la evolución y reconstrucción de la sociedad, de tal manera que se convierta en otra más digna y justa, con un desarrollo económico y social fundamentado en la libertad, la independencia, la cooperación, la rectitud moral y la responsabilidad social.

\section{Palabras clave}

Estudios Generales, Ciclo Básico, formación integral, humanismo cristiano, Pontificia Universidad Católica Madre y Maestra

\section{Key Words}

General Studies, Basic Education Term, comprehensive education, Christian humanism, Pontificia Universidad Madre y Maestra
En vista de lo antes expresado, la universidad, como institución que participa en la transformación de la sociedad, debe cuestionarse cuáles conocimientos deben poseer sus egresados. Según Luis Bernardo Peña (1986), debemos entender que "El conocimiento es aquel que trasciende las fronteras de las profesiones y de lo utilitario, el conocimiento común que constituye una parte importante del tejido de una cultura; aquello que debiera permanecer cuando los aprendizajes particulares se olviden o pierdan vigencia".

Esto nos lleva a pensar en el rol que debería desempeñar la universidad. Trottini y Marrengo (2007) afirman que la función prioritaria de la universidad de hoy está dada por la conservación del patrimonio cultural e histórico de una sociedad y el logro de las transformaciones nece-

\begin{abstract}
Licenciada en Psicología por el Instituto Tecnológico de Santo Domingo. Especialidad en Técnicas de Evaluación e Intervención Psicoeducativa para Contextos Escolares y Sociocomunitarios por la Universidade da Coruña, España y la Pontificia Universidad Católica Madre y Maestra. Directora del Departamento de Ciclo Básico de esta última universidad en el Recinto Santo Tomás de Aquino. Para contactar a la autora: LuisaTaveras@pucmm.edu.do

“*icenciada en Educación, Mención Letras por la Pontificia Universidad Católica Madre y Maestra, Campus de Santiago. Magíster en Investigación Educativa por la Universidad Tecnológica de Santiago. Docente del área de Letras en el Departamento de Humanidades de la PUCMM, Campus de Santiago. Para contactar a la autora: rolivo@pucmmsti.edu.do
\end{abstract}

Encuentre el texto en "Los Estudios Generales en el currículum universitario" http://www.pucmm.edu.do/STI/campus/CDP/ComunicaciónPublicaciones/Paginas/CuadernodePedagogiaUniversitaria.aspx Cuaderno de Pedagogía Universitaria Año 7/ N. 14 / julio - diciembre 2010 / Santiago, Rep. Dom.: PUCMM / pp.25-29 
sarias para adecuarse a las demandas del mundo actual. Aseveran que la Educación Superior debe tener mayor capacidad de respuesta a los problemas generales con que se enfrenta la Humanidad y las necesidades de la vida económica y cultural. Además, la enseñanza debe ser más pertinente en el contexto de los problemas específicos de una región, un país o una comunidad. Así mismo, exponen que este planteamiento involucra una cantidad de desafíos que se deben enfrentar y superar, tales como: en primer lugar, por medio de los procesos de capacitación, lograr contribuir con la organización social a fin de fortalecer la sociedad civil incrementando los niveles de educación, de alimentación; además de proteger el medio ambiente, respetar la ley y acatar la justicia. Segundo, debemos enmarcar toda actividad que conduzca a promover el desarrollo humano sostenible. Por último, tenemos que denunciar toda acción que involucre actos de impunidad y de inmoralidad, con la finalidad de fomentar una sociedad basada en valores.

Estos desafíos exigen un cambio de visión en el proceso de formación de los profesionales. Este cambio de visión no puede reducirse a cambiar lo defectuoso, implica creación. Tendríamos que abocarnos a una reforma universitaria donde se reformule la misión de la universidad, dar respuesta a la pregunta de Ortega "¿para qué existe y tiene que estar la Universidad?"

Ramsden (1995) cita al Cardenal Newman, quien afirma que la universidad "Es un lugar para la enseñanza del conocimiento universal, su objeto, es la difusión y la extensión del conocimiento más que su desarrollo. Si su objeto fuera el descubrimiento científico y filosófico, no veo por qué una universidad debería tener estudiantes".

Al analizar la historia, nos damos cuenta que tal fue la orientación de la universidad en la Edad Media. Según Ortega, la Universidad medieval no investigaba, se ocupaba muy poco de la profesión en sí, todo era cultura general. Lo que hoy se llama confusamente "cultura general" no lo era para la Edad Media. La cultura general de la que hoy se habla es percibida como un adorno de la mente o disciplina del carácter. En los tiempos medievales, esta cultura general, por el contrario, era vista por Ortega como el sistema de ideas sobre el mundo y la humanidad, que el hombre de entonces poseía. Era, pues, el repertorio de convicciones que había de dirigir efectivamente su existencia. Ortega ve la vida como una selva en la que el hombre se pierde, pero en la que trabaja para encontrar caminos, es decir, ideas claras y firmes sobre el Universo, convicciones positivas sobre lo que son las cosas y el mundo. Estas ideas tenían un valor fundamental para Ortega, pues para él nosotros somos nuestras ideas.

En el pensamiento orteguiano la misión que le corresponde a la universidad es la reconstrucción de la cultura, y esta no puede conseguirse sin una formación de carácter general. Expresa que el profesionalismo y el especialismo dominantes en la universidad moderna han roto en pedazos al hombre y han acelerado la fragmentación de la cultura y ve como necesario armar el rompecabezas y reconstruir con los pedazos dispersos la unidad fundamental del hombre. En esta tarea le corresponde a la universidad una misión fundamental.
Al igual que Ortega, Hutchins criticó el especialismo y su secuela: la fragmentación del conocimiento.

Es función de la universidad mantener y desarrollar la cultura de la sociedad a través de la formación de personas que se apropien de ella, la apliquen y la enriquezcan y, de esa manera, logren llegar a la solución de los problemas con un enfoque crítico, reflexivo e innovador. Si bien es cierto que la sociedad necesita buenos profesionales (médicos que curen, maestros que enseñen, abogados que defiendan) también necesita, como dice Ortega, profesionales que sean capaces de vivir e influir vitalmente según la altura de los tiempos y, por eso, se hace necesario crear en la universidad la enseñanza de la cultura o sistema de las ideas vivas que el tiempo posee. Nuestra sociedad está llena de profesionales, "bárbaros especialistas" recluidos en su propia esfera de conocimiento, que gloriosamente contribuyen al progreso de la ciencia, pero que en muchas otras ocasiones, amparados en su limitada sabiduría, actúan de manera petulante en el resto de parcelas del saber (en política, arte, en los usos sociales, en otras ciencias, etc.).

Cuando la universidad no hace del hombre, un hombre culto, capaz de transformar su mundo, las actuaciones de este, al trascender su oficio, resultarán deplorables (tristes, angustiosas, lamentables, patéticas, dolorosas). Citamos textualmente a Ortega al referirse al profesional sin cultura general: "Sus ideas y actos políticos serán ineptos, sus amores, empezando por el tipo de mujer que preferirá, serán extemporáneos y ridículos, llevará a su vida familiar un ambiente inactual, maniático y mísero que envenenará para siempre a sus hijos y, en la tertulia del café, emanará pensamientos monstruosos y una torrencial chabacanería". Además de buenos profesionales, necesitamos una ciudadanía inteligente, así la llamó Hutchins.

Sin duda alguna, esto nos lleva a afirmar que la educación influye decisivamente en el progreso de una comunidad y se hace inevitable para la conservación y desarrollo de su cultura. Es deseable que todo profesional universitario, independientemente de su área de especialización, adquiera un conocimiento amplio y un sentido vivo de los valores fundamentales que conforman la cultura en la cual se desarrolla. Luis Bernardo Peña (1986) entiende que hay que "devolver a la universidad su tarea central de ilustración del hombre, de enseñarle la plena cultura del tiempo, de descubrirle con claridad y precisión el gigantesco mundo presente, donde tiene que encajar su vida para ser auténtica".

En consecuencia, el núcleo del currículum universitario deberá estar conformado por la Educación General como garantía de darle sentido a la formación especializada y que el hombre pueda valorar su vida desde una óptica individual y social. Ortega y Hutchins enfatizaron que en las universidades debería haber una instancia que se ocupara de la Educación General. Ortega la llamó la Facultad de la Cultura y Hutchins, el College.

Todas las universidades dominicanas, por norma, deben contemplar en sus planes de estudios la Educación General, ya que así queda

Estudios Generales: el qué y el para qué. Apuntes para una reflexión, Luisa Taveras y Rosario Olivo

Cuaderno de Pedagogía Universitaria Año 7/ N. 14 / julio - diciembre 2010 / Santiago, Rep. Dom.: PUCMM / pp.25-29 
establecido en el artículo 11 del Reglamento para Evaluación y Aprobación de Carreras a Nivel de Grado de la Secretaría de Estado de Educación Superior Ciencia y Tecnología, hoy Ministerio.

En ese artículo se establece que las carreras de grado se deben organizar en una estructura curricular de por lo menos dos ciclos o bloques, que pueden ser denominados Ciclo de Formación General y Ciclo de Formación Especializada. El Ciclo de Formación General está orientado al desarrollo integral, humanístico, social, ético y tecnológico, mediante la adquisición de competencias, actitudes y valores que permitan alcanzar una visión general del mundo, incrementar la imaginación, la innovación y la criticidad favorables a la autonomía y a la educación permanente. El Ciclo de Formación Especializada está dirigido al desarrollo de competencias, actitudes y valores que preparan para un ejercicio profesional exitoso. Este ciclo puede subdividirse en el Ciclo de Formación General del Área y Ciclo de Formación Especializada, propiamente dicho.

Es interesante destacar que el párrafo I de dicho reglamento establece que la estructura curricular en ciclos es un indicativo de las opciones de organización que cada institución dará a la oferta curricular conforme a su naturaleza, misión, objetivos y perfiles de egreso de los profesionales formados.

Esta libertad que se da en ese párrafo lleva a que cada institución utilice diversas denominaciones para designar los primeros años de la vida universitaria. Para nombrar este primer ciclo de la Educación Superior se han utilizado términos como Ciclo Básico, Propedéutico, Preuniversitario, Colegio Universitario. Esto, sin duda, es un reflejo de la falta de consenso existente en cuanto al significado e importancia de los Estudios Generales en el currículum universitario. Algunas instituciones lo ven como base para estudios especializados y profesionales y como inherentes a la formación universitaria y otros consideran que su función es remediar las deficiencias de la Educación Media.

\section{Quintero (s.f.) afirma que:}

"La Educación General debe verse desde varios puntos de vista. Desde el punto de vista de la persona, es aquella educación que encamina al estudiante hacia el logro mayor de sus capacidades de expresión, reflexión apreciación, deliberación y convivencia. Desde el punto de vista del saber, introduce al estudiante en el examen crítico de las mejores situaciones que ofrecen las Ciencias Sociales, las Humanidades y las Ciencias Naturales. Ambos aspectos se interrelacionan, ya que el saber es la expresión del hombre y, por lo tanto, formativo de los hombres. La persona y el saber se dan en un contexto social, en nuestro caso, en una sociedad en rápida transformación. Este contexto afecta las actitudes y motivaciones de estudiantes y maestros. De estas realidades hay que partir, no meramente como dato para responder pasivamente, sino pensando en un ideal de persona y de sociedad. Por lo tanto, toda definición de Educación General está estrechamente ligada al concepto de libertad humana. En nuestro caso, el ideal es la persona libre en una sociedad libre, aquella que propulsa el desarrollo progresivo de las facultades de expresión, re- flexión y deliberación. En estas definiciones Educación General y democracia están imbricadas profundamente."

Este mismo autor, en su escrito “Educación general: propósitos, métodos, contenidos" (s.f.) plantea tres objetivos principales para los estudios generales:

“primero, proveer aquellos conocimientos básicos necesarios a todas las profesiones, es decir, conocimientos mínimos del lenguaje, matemáticas, historia, geografía y ciencias sobre los cuales se monta el resto de la educación. En segundo lugar, la comunicación cívica, o sea, la capacidad de entender las diversas esferas de acción en la sociedad y de comunicación y apreciación entre ellas. Por último, el desarrollo de la persona".

Este autor afirma que para lograr este objetivo, la finalidad, el método educativo y el contenido de la educación se regirán por un mismo principio. El progreso de la persona se medirá a base del desarrollo de las artes, humanidades, la ciencia y la política: las artes del análisis, apreciación, comunicación y deliberación. El contenido de la educación estará compuesto por aquellos problemas, documentos y ejemplos que ilustran estas artes y el método será la práctica continua de las mismas.

Manuel Maldonado Rivera (1983) expresa que "la Educación General es lo que la institución entiende que todos sus estudiantes, en la sociedad y en un momento histórico determinado, deben aprender". Con relación a los objetivos de la misma, señala que:

"primero, la Educación General deberá continuar con el desarrollo de los niveles superiores de aquellas habilidades, destrezas y actitudes que consideramos distintivas de la especie humana y cuyo perfeccionamiento posibilita una realización más plena de lo humano, una ampliación de la capacidad del individuo para tener experiencias significativas, para vivir una vida digna, libre y liberadora. Segundo, la Educación General debe propiciar que el estudiante explore y comprenda las modalidades fundamentales del conocimiento. Tercero, la Educación General debe promover en el estudiante una clara conciencia de la función vital del conocimiento, de la pertinencia de las disciplinas fundamentales para la vida real, del poder que nos da el conocimiento para transformar la realidad. Cuarto, la Educación General no debe limitar sus expectativas a desarrollar una persona, a formar la persona en abstracción de la realidad social en la que realiza su existencia. Quinto, la Educación General tiene que promover la discusión crítica y concientizar al estudiante de que tiene un compromiso con un mundo mejor. Sexto y último, la Educación General debe propiciar en sus cursos una metodología de la enseñanza y un espíritu de solidaridad y búsqueda compartida en el ambiente humano en que se desenvuelve".

Para Estrella Guerra Caminiti (2010) los actuales Estudios Generales implican el estudio de las Humanidades, pero también de otros saberes como son las Ciencias Sociales, las Ciencias Naturales y Exactas, y, especialmente, brindar las posibilidades de lograr un nivel más que satisfactorio en lo que es la redacción, la argumentación y la investigación. Esto significa claramente que se trata de 
ofrecer una formación multidisciplinaria. Sin embargo, para esta autora, el currículum esbozado no es suficiente para cumplir con el objetivo propuesto. Afirma que no se trata tan solo de asegurar el conocimiento, reflexión y práctica en un determinado número de materias, sino que es muy importante que existan características que aseguren que la educación que brinden las diversas asignaturas logrará la formación de ciudadanos libres y democráticos.

En este punto Guerra Caminiti (2010) sigue lo propuesto por Martha Nussbaum en su libro "El cultivo de la humanidad" (2001) como las características básicas de una educación humanista, que ella define como liberal, que deben ser la impronta en la que se imparten los estudios disciplinarios.

Nussbaum propone como una de estas características la educación estimulante e incisiva que despierta el sentido crítico de los estudiantes y los ayuda a discernir con lucidez. Señala que el estudio de la Filosofía es especialmente útil para comprender cómo conocemos, cómo construimos nuestras valoraciones, etc. Guerra Caminiti agrega que para lograr esta meta resultaría muy útil el estudio y práctica de la Argumentación, entendida como la antigua Retórica.

Una segunda característica propuesta por Nussbaum sería la de promover una mirada intercultural, es decir, el estudio de otros grupos humanos, otras lenguas y otras costumbres, además de la propia. Esta es la manera de promover en los estudiantes una reflexión crítica sobre la propia cultura y desarrollar en ellos la conciencia de la importancia de la dimensión política. En este sentido, disciplinas como las Ciencias Sociales, la Lingüística, la Literatura, la Historia, la Filosofía pueden aportar con sus reflexiones, cooperando en la comprensión de otras culturas, sin olvidar dentro de ellas a los grupos minoritarios y colaborando en su comprensión.

La tercera característica mencionada por la autora es la que se refiere a desarrollar una "imaginación narrativa". Con esta expresión alude a la capacidad que nos permite ponernos en el lugar del otro $y$, en ese encuadre particular, construir la narración que me permita experimentar lo que le pueda estar aconteciendo. Es el terreno de la empatía que, sin perder la distancia crítica que me permite conocer, me brinda la oportunidad de sentir con el otro. Para desarrollar esta capacidad son especialmente interesantes los cursos artísticos que cultivan la sensibilidad.

Finalmente, Nussbaum sugiere la importancia de integrar a la formación liberal el saber científico que aporta la reflexión de las ciencias naturales y de las ciencias exactas que contribuyen con la comprensión del valor de la abstracción y de los procesos inductivos y deductivos en la construcción de cualquier forma de conocimiento.

Guerra Caminiti (2010) afirma que "Ios Estudios Generales tienen un fin en sí mismos, que ciertamente se proyecta hacia la sociedad, pero que no debe cumplir con una función remedial de subsanar aquello que no se logró con la formación escolar, ni tener una dimensión propedéutica de preparación para la especialización".
En este punto es importante destacar dos ideas cardinales, primero, la formación general no debería ser misión exclusiva de la universidad, porque no podemos pretender lograr este objetivo tan ambicioso en un período tan corto. Esta formación debería ser tarea de todo el sistema educativo, tanto la Educación Básica como la Educación Media.

Segundo, no podemos caer en el error de pensar que el simple paso del estudiante por una serie de cursos de Estudios Generales va a configurar el ser humano intelectual y ético que nuestra sociedad necesita. En este sentido, es trascendental destacar que no es solo el contenido del curso propiamente, sino también la óptica pedagógica con el que el mismo se imparte como proceso. Por ejemplo, un curso sobre Física puede despertar un debate que trascienda las fronteras de esta área, sobre una problemática que no sea intracientífica. La mayoría de veces, en estos cursos, lo que se busca pedagógicamente es que el alumno maneje o almacene la mayor cantidad de datos o informaciones posibles, reduciendo así las posibilidades del alumno para asumir una posición crítica sobre un problema de su sociedad que le atañe, le afecta y ante el cual pudiera proponer una solución. Asusta pensar que, finalmente, lo que la educación está haciendo es poner a andar las enciclopedias.

Así mismo, en estos cursos se estudia la ciencia sin relacionarla o entenderla en el contexto en la que se desarrolla, se estudia con una visión seccionada, analítica y estática. Un curso de Estudios Generales debe superar esto. No podemos comprender el psicoanálisis de hoy sin entender la teoría freudiana enmarcada en la clásica, respetuosa y conservadora burguesía de Viena. Los cursos de Estudios Generales deben ver la ciencia desde un punto histórico- evolutivo.

Desde la óptica pedagógica, un curso de Estudios Generales busca que el estudiante viva una serie de experiencias conducentes a la formación integral, donde confluyen conocimientos, destrezas, habilidades, competencias genéricas y específicas, actitudes y valores fundamentados en el amor, la dignidad humana, la libertad y la justicia social. En este proceso se privilegiará el aprendizaje, el centro no será ni el alumno ni el profesor sino el conocimiento. Este se construirá a partir de la relación de estos protagonistas en el contexto educativo.

En este sentido, concebimos el conocimiento como un proceso que implica hacer uso de la reflexión, la problematización, la creación y la comunicación en la elaboración de saberes. De tal modo, que no se insiste en los contenidos o las materias con el fin de que el alumno tenga un cúmulo de saberes enciclopédicos; por el contario, se evita la memorización mecánica como método de enseñanza, ya que finalmente, esa memorización en vez de formar, deforma al estudiante como ente pensante y lo que produce es un ciudadano dogmático.

Entendemos que la Educación General ofrece un campo de experiencia dirigida a la formación integral a través de enfoques multi e interdisciplinarios construidos a partir de diversas áreas del conocimiento. Es un nivel formativo, no remedial, donde el educando aprende a conocer, a hacer, a aprender, a ser, a conducirse en la vida y a trascender. Se caracteriza por ser un proceso dinámico donde 
se examinan los fundamentos y procesos de producción del conocimiento, el contexto histórico y cultural.

La Pontificia Universidad Católica Madre y Maestra (PUCMM), está consciente de que la misión de la universidad es formar a un ser humano que tenga una comprensión fundamental del mundo en que vive, con un razonamiento moral y juicio ético, que entienda las claves del proceso histórico y que posea una visión reflexiva y crítica de la propia cultura y las culturas del mundo. Por eso, la PUCMM ha iniciado un proceso de revisión, reflexión y reforma del Ciclo Básico.

La reflexión está partiendo de que los Estudios Generales no están destinados a ser remediales. Deben estar enfocados en uno o pocos temas, por lo tanto, nunca un curso introductorio debe formar parte de los Estudios Generales. También, estos cursos deben articularse en torno a la investigación, a la lectura, el laboratorio, el debate, es decir, a la producción de parte del estudiante y los profesores.

También se está considerando que estos cursos deben ofrecer la compresión de los conceptos claves de cada disciplina, así como analizar y examinar los diferentes métodos para la construcción del conocimiento. Además, deben mostrar y explorar los vínculos de una disciplina determinada con otras y establecer relaciones entre los saberes de las diferentes disciplinas que aportan al conocimiento en cada área de estudio. En este orden, se busca vincular y valorar todos los saberes concernientes a la persona, desde una perspectiva del humanismo cristiano.

Es deseable que esta reforma contemple promover en el estudiante una actitud inquisitiva, reflexiva y dialogante, por lo tanto, el enfoque de estos cursos debe ser participativo y crítico. Además, promover el logro de resultados donde los estudiantes puedan presentar a sus compañeros de clase u otros, en forma de conferencias, exposiciones, artículos, etc., los aprendizajes de los diferentes cursos. La oferta de cursos debe ser flexible para que puedan articularse propuestas de temas actuales, siempre que haya uno o varios docentes involucrados en su investigación.

La propuesta de reforma del Ciclo Básico se encuentra en proceso de reflexión, revisión, autocrítica y aún después que se implemente sabemos que esto será un espiral, no se quiere establecer un canon de Estudios Generales o asumir el modelo de una determinada institución, como dice Ortega "No importa si llegamos a las mismas conclusiones y formas que otros países, lo importante es que lleguemos a ellas por nuestro propio pie" a través del personal combate con las ideas.

En este proceso se han detectado necesidades como es la formación y el acompañamiento continuo de los maestros así como cambios que hacer en la estructura y organización de la Universidad. En fin, tenemos un trabajo arduo por delante, pero estamos convencidos del mismo. Kant dice: "es provechoso tratar al hombre conforme a su dignidad, puesto que es algo más que una máquina". Son los Estudios Generales que harán posible esto, apoyamos esta idea.

\section{Referencias bibliográficas}

Guerra Caminiti, E. (2010) La importancia de la multi e interdisciplinariedad en los Estudios Generales: El caso de los Estudios Generales Letras de la Pontificia Universidad Católica del Perú. Ponencia presentada en el V Coloquio Internacional de Educación General. Universidad Autónoma de Santo Domingo, abril 2010.

Hutchins R.M. (1995). The Higher Learning in America. New Brunswick: Transaction Publishers.

Kant, I. (1978). ¿Qué es la llustración? En Filosofía de la Historia, Buenos Aires: Ed. Nova.

Maldonado Rivera, M. (1983). El concepto de educación general en Maldonado. En: Rivera, M. (Ed.) La educación general y la misión de la Universidad de Puerto Rico. San Juan: Universidad Interamericana de Puerto Rico.

Nussbaum, M.C. (2001). El cultivo de la humanidad. Una defensa clásica de la reforma en la educación liberal. Santiago de Chile: Editorial Andrés Bello.

Ortega y Gasset, J. (1983). Misión de la universidad, Obras Completas, 1a. ed., Tomo IV. Madrid: Alianza Editorial

Ortega y Gasset, J. (1986). La rebelión de las masas. Madrid: Alianza Editorial

Peña, L.B. (1986). Vigencia de la Educación Liberal en el Currículo de la Universidad. Conferencia XXIV, Simposio Permanente sobre la Universidad, Bogotá (1985-1987)

Quintero Alfaro, A. G. (S.F.) ¿Que es la educación general? Mimeografiado. Facultad de Estudios Generales, Recinto Río Piedras de la Universidad de Puerto Rico.

Quintero Alfaro, A. G. (S.F.) Educación general: propósitos, métodos, contenido. Mimeografiado. Facultad de Estudios Generales, Recinto Río Piedras de la Universidad de Puerto Rico.

Ramsden, P., Margetson, D., Martin, E., \& Clarke, S. (1995). Recognising and rewarding good teaching in Australian higher education: Griffith Institute of Education, Griffith University.

Trottini, A.M. y Marengo, H. (2007) ¿Cuál es la función de la Educación Superior en la sociedad actual? Ponencia presentada en las II Jornadas Nacionales y I Latinoamericanas de Pedagogía Universitaria "Enseñar y aprender en la Universidad" "Culturas y educación en la Universidad: problemas y perspectivas" Universidad Nacional San Martín. San Martín, Prov. de Buenos Aires, Argentina - 6 y 7 de septiembre de 2007. 